
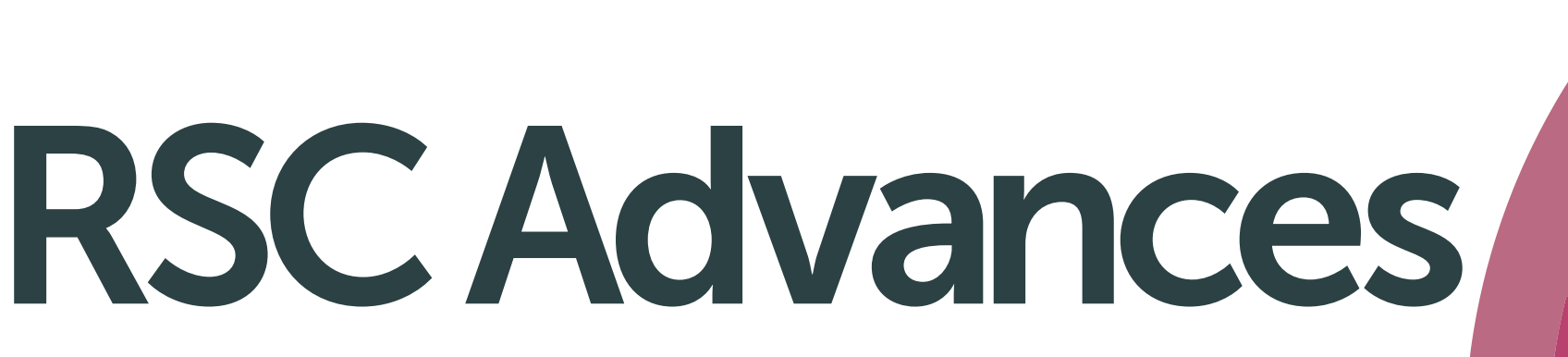

Volume1 Number1 Jan 2013 $\mid$ Pages 1-100

\section{RSC Advances}

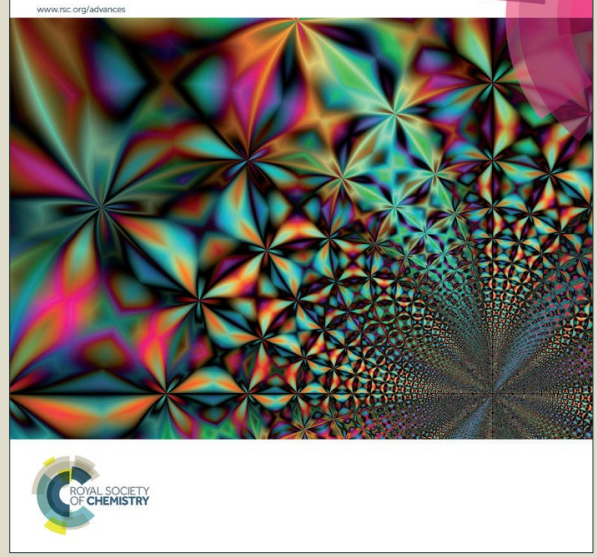

This is an Accepted Manuscript, which has been through the Royal Society of Chemistry peer review process and has been accepted for publication.

Accepted Manuscripts are published online shortly after acceptance, before technical editing, formatting and proof reading. Using this free service, authors can make their results available to the community, in citable form, before we publish the edited article. This Accepted Manuscript will be replaced by the edited, formatted and paginated article as soon as this is available.

You can find more information about Accepted Manuscripts in the Information for Authors.

Please note that technical editing may introduce minor changes to the text and/or graphics, which may alter content. The journal's standard Terms \& Conditions and the Ethical guidelines still apply. In no event shall the Royal Society of Chemistry be held responsible for any errors or omissions in this Accepted Manuscript or any consequences arising from the use of any information it contains. 
Table of contents:

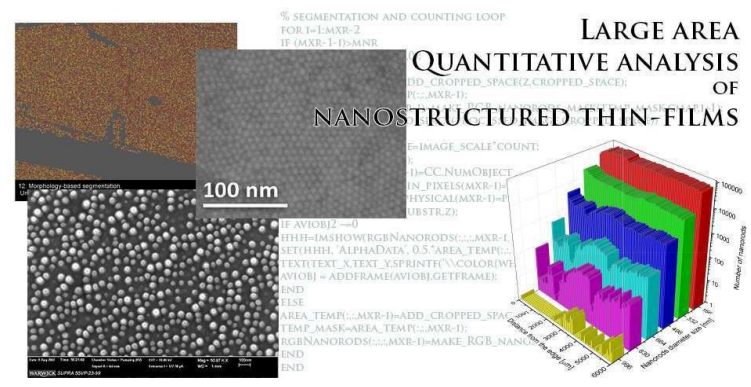

Proposed and verified method offers an unique quantitative large scale nanostructures' evaluation 


\title{
Large area quantitative analysis of nanostructured thin-films
}

\author{
Rafal Sliz, ${ }^{*}{ }^{\ddagger}$ Chibuzor Eneh, ${ }^{a \ddagger}$ Yuji Suzuki,,${ }^{b \ddagger}$ Jakub Czajkowski, ${ }^{a \ddagger}$ Tapio Fabritius, ${ }^{a}$ Poopathy \\ Kathirgamanathan, ${ }^{c}$ Arokia Nathan, ${ }^{d}$ Risto Myllyla $^{a}$ and Ghassan Jabbour ${ }^{e}$
}

\author{
Received Xth $X X X X X X X X X X 20 X X$, Accepted Xth $X X X X X X X X X 20 X X$ \\ First published on the web Xth $X X X X X X X X X X 200 X$ \\ DOI: $10.1039 / b 000000 x$
}

1-D nanostructured thin-films exhibit, amongst other properties, unique mechanical, electrical, thermal, and optical properties. These depend strongly on several aspects, including size, dimension and density. A thorough characterization of a nanostructured film requires extensive time and is a great effort in terms of human resources. This article presents a facile implementation of an automatic quantitative method for the characterization of nanostructured thin-films using a SEM image-based automatic characterization solution to evaluate the size distribution and surface area (areal density) of assembled structures on a large scale. The implemented solution has been used to evaluate electrochemically deposited zinc oxide nanorod thin-films as well as additional inorganic thin-films. To validate the results, the proposed characterization method was compared with manual small-scale characterization methods.

\section{Introduction}

The miniaturization of electronics to the submicron scale allows the electronics industry to develop devices with a high scale of complexity at reduced cost. Nonetheless, the current advance in electronics requires further development/improvements in terms of miniaturization and performance. One possible solution is diverse one-dimensional (1D) nanostructures, which have been manufactured from a wide range of materials. Nanostructures are important due to their high surface-to-volume ratio and their influence on the electrical, optical and mechanical properties of the fabricated films. Some examples include nanorods, nanowires and nanobelts, based on polymers, metals and semiconductors. Some of these newly emerging materials are forecasted to enable novel functions and enhanced performance ${ }^{1-7}$. Widely spread semiconductor nanostructures with efficient electron and exciton transport properties are a promising material for nanoscale electronics, especially for optoelectronic devices ${ }^{8-13}$. Since small changes in the properties of nanostructures have a great influence on device performance, overall device reliability depends heavily on the consistency of the used nanostructures. For ex-

\footnotetext{
a Optoelectronics and Measurement Techniques Laboratory, University of Oulu, 90570 Oulu, Finland. Fax: +358 8553 2774; Tel: +358 0294482 769; E-mail: rafal.sliz@ee.oulu.fi

${ }^{b}$ London Centre for Nanotechnology, University College London, 17-19 Gordon Street, London WC1H OAH, United Kingdom

c Organic Electronics, Wolfson Centre, Brunel University, Uxbridge UB8 3PH, United Kingdom

d Centre for Advanced Photonics and Electronics, Cambridge University, 9 JJ Thomson Avenue, Cambridge CB3 OFA, United Kingdom

e Renewable Energy Center, University of Nevada, Reno, NV 89557-0388, USA

$\ddagger$ These authors contributed equally to this work.
}

ample, the morphology (areal density and diameter size distribution) of nanostructures strongly affects the properties of thin-films. As a result, quantitative characterization is crucial for fabricated nano-enhanced thin-films ${ }^{14}$.

The most common methods used to evaluate nanostructure morphologies are Atomic Force Microscopy (AFM), Scanning Electron Microscopy (SEM) and Transmission Electron Microscopy (TEM), to name a few. Although these methods provide high quality measurements, the time and human resources that they demand restricts the total area that can be scanned. Moreover, significant errors may arise from nonuniform sample locations. In addition, data produced by these methods tends to be quantitative only locally and can be misinterpreted for larger scale thin-films. Several researchers have addressed the characterization problem to perform a quantitative analysis of nanostructured thin-films. For instance, some groups have proposed a simple algorithm to count particle size distribution based on SEM and TEM images ${ }^{15,16}$. Also, some studies describe a particular procedure to determine the areal density of nanostructures from electron micrographs at different locations and magnifications by manual counting ${ }^{17,18}$. More comprehensive studies on TEM images have been conducted by Mondini et al., although their methods were based on single images of one area of interest ${ }^{19}$. Additionally, biologists developed multiple mechanisms and algorithms to produce quantitative information regarding the number of cells in the tissue, cell size and much more ${ }^{20}$. Nonetheless, the large area thin-film analysis still remains an unexplored field.

This paper proposes a new quantitative approach for areal density analysis and size distribution characterization of nanostructures. The method proposed here is a program that implements image-based morphological operations in 
a unique manner to assess SEM images of nanostructureenhanced thin films ${ }^{21}$. Moreover, the presented program has image enhancing features, which significantly improve the quality of acquired images. In this experiment, zinc oxide nanorods ( $\mathrm{ZnO} \mathrm{NRs}$ ) were selected as main research subject for their high applicability in optoelectronics ${ }^{22-24}$. In addition, self-assembled gold quantum dots and InP-based nanostructures were examined. Prior to the analysis, $\mathrm{ZnO}$ fabricated thin-films were additionally characterized with AFM and XRay Diffraction (XRD) to acquire information on the dimensions and orientation of nanostructures. A series of SEM images was acquired and the proposed program based on the developed algorithms was used to analyze the data. This allowed the extraction of the areal density and size distribution of nanostructures. Finally, to evaluate the program usability, achieved data was manually verified.

\section{Methods}

\subsection{ZnO nanorod fabrication}

Zinc oxide NRs were used to verify the developed algorithm. $\mathrm{ZnO}$ NRs were electrochemically deposited from 50 $\mathrm{mM}$ aqueous zinc nitrate (ZnNO) solutions (Sigma Aldrich, $99.999 \%$ ) on ITO-covered glass substrates (TFD, resistivity $20 \Omega / \square$ ). The galvanostatic deposition method with undivided three-electrode cell arrangement was adapted from Seipel et al. and D'Alkaine et al. ${ }^{25,26}$. For $45 \mathrm{~s}$, pre-cleaned ITO (working electrode) was halfway immersed in the zinc nitrate solution (Fig. 1(a)), at a distance of $2 \mathrm{~cm}$ from a $1 \mathrm{~cm}^{2}$ platinum foil (auxiliary electrode). A standard calomel electrode $\left(\mathrm{E}_{0}\right.$ $=+240 \mathrm{mV}$ ) was used as reference. The potential with respect to the reference electrode was $650 \mu \mathrm{A} / \mathrm{cm}^{2}$. Moreover, growth parameters were controlled and monitored with the potentiostat/galvanostat PAR 270. For all experiments, solution temperature and acidity were maintained at $80{ }^{\circ} \mathrm{C}$ and 5.3 , respectively.

\subsection{Physical characterization and imaging of fabricated films}

To determine the dimensions and orientation of NRs, the fabricated films were initially characterized using AFM (Veeco D3100 AFM) and XRD (Philips X'pert Theta2Theta, $\mathrm{Cu} \mathrm{K} \alpha$ $(\lambda=1.54 \AA)$, power $=40 \mathrm{kV} 50 \mathrm{~mA})$. X-rays were used to analyze the crystallographic orientation of the fabricated nanostructures. As presented in Fig. 1(c), AFM inspection was performed in three different locations of the sample. The same figure shows how $\mathrm{ZnO}$ NRs were inspected by SEM imaging (JEOL JCM-5000) using an imaging area of 85 x $100 \mu \mathrm{m}$ (real sampling area). This inspection was performed from top to bottom starting from the air/solution edge, including the area

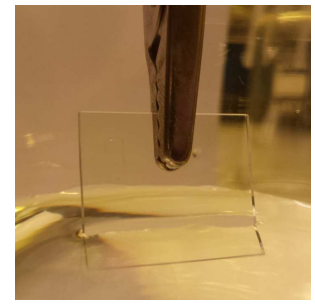
immersed in the zinc nitrate solution (a) Image of ITO sample

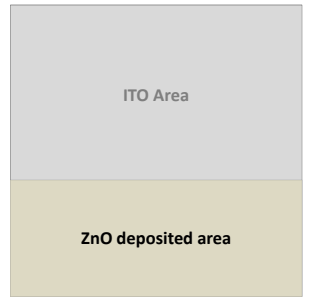

(b) Area covered by $\mathrm{ZnO}$ nanorods after deposition

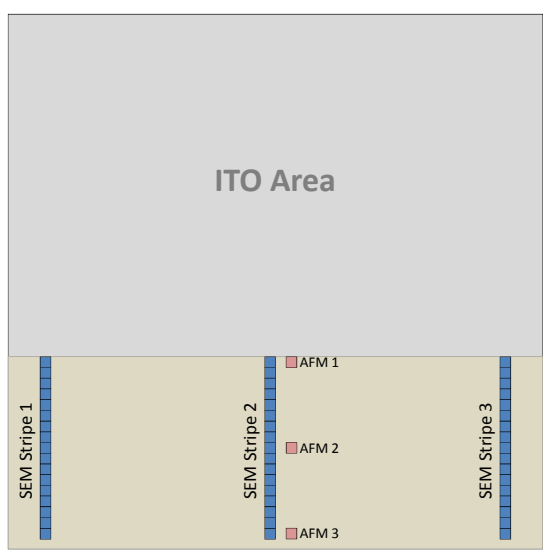

(c) Location of SEM and AFM images taken for surface characterization

Fig. 1 Depicted ITO-covered glass sample and location of SEM and AFM inspection areas.

where the NRs begin to form. In this regard, three $6 \mathrm{~mm}$ long strips of ZnO NR SEM images were taken and analyzed.

\subsection{Program implementation}

A Matlab-based computation algorithm was implemented to post-process SEM images and to quantitatively determine the properties of $\mathrm{ZnO}$ NR films. The algorithm comprises two stages. In the first stage, each SEM image is enhanced and filtered to exclude all major artefacts. In the second stage, the post-processed image undergoes a morphology-based segmentation to calculate areal density and NR size distribution. To make the algorithm steps easy to understand, diagrams in Fig. 2 and Fig. 3 demonstrate the image enhancement and NR recognition processes. Importantly, the program automatically adapts enhancement parameters individually to each image, resulting in better NR recognition and ambiguity-free results. Detailed processing steps regarding animation and source code are provided in supplementary information.

Processing starts with the loading of SEM images (step 1) into a memory buffer. Each image is then cropped (step 2) to exclude the embedded SEM parameter caption. Next, the im- 


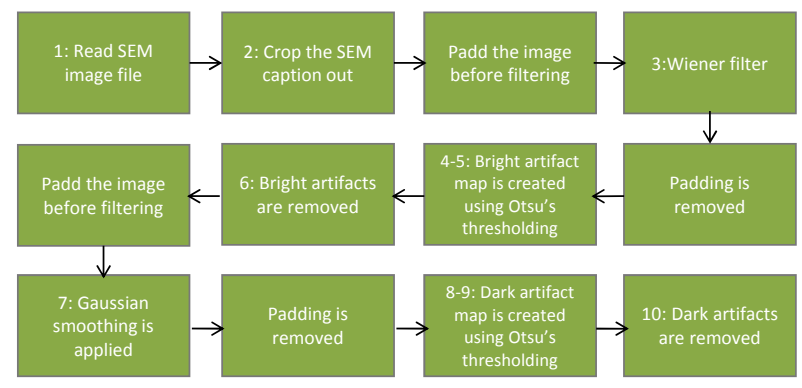

Fig. 2 SEM image artefact removal and enhancement.

age is padded with 128 pixels on each side to suppress edgerelated artefacts resulting from the filtering processes. This prepared image is subjected to a Wiener filter (step 3), which is a trade-off between speed and accuracy, with a kernel size of $24 \times 24$ pixels ${ }^{27}$. In this application, the filter exaggerates bright objects and smooths out the surroundings, allowing the pin-pointing of such artefacts as dust particles. After filtering, the padding is removed to revert the size of the image to its original size. The filtered image is binarized (step 4) using a thresholding algorithm based on the Otsu method ${ }^{28}$. The resulting binary map serves as a mask, used to exclude artefacts. At the next step, all bright artefact features are removed (step 6). However, to increase the accuracy of quantitative analysis, all dark features are also investigated, since their presence may indicate a scratch, for example. To create a map of dark artefacts, the image corrected for bright artefacts is morphologically dilated to fill the areas between NRs and preserve all significant dark spaces. It is then padded with 128 pixels using Wiener filtering, in the same way as before, and then Gaussian filtered with a $16 \times 16$ pixel kernel (step 7). The padding is subsequently removed and the image is binarized using a thresholding algorithm (step 8-9). As previously, a mask that excludes all significant dark artefacts from the image is created (step 10). Both computed artefact masks are applied to the original image, and areas occupied by artefacts are filled with a value corresponding to the median of the image. These maps are saved for later use to analyze NRs.

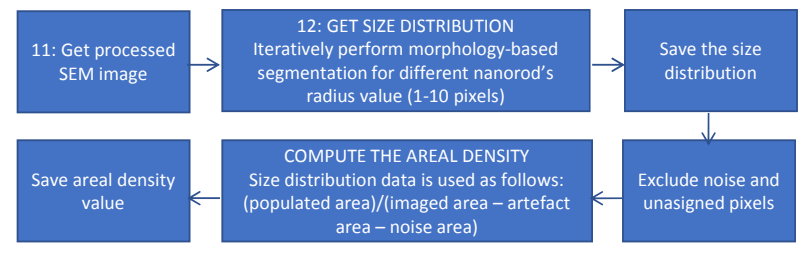

Fig. 3 Nanorod counting and areal density computation.

A quantitative study of NR films is presented in Fig. 3 and consists of post-processed images that are morphologically segmented in a loop, using circular features with decreasing radius. In each loop step, the segmented fraction is subtracted from the data, so that only smaller features are present in the image at the following step. When the loop converges, a complete size distribution of NRs is available. This data is subsequently used to determine the areal density of the NR film. The computation can be described with the following equation:

Areal density $=\frac{100 \cdot \text { Size of cropped image }}{(\text { Sumof classified nanorod areas }- \text { Artifact areas-Unclassified areas })} \%$

\subsection{Verification Mechanisms}

To assure the reliability of results provided by the developed software, verification was performed. The SEM image presented in Fig. 4 was used for verification purposes. Firstly, the number of NRs was counted by two human subjects, a commercial software package (Nikon NIS-Elements BR6.2 ${ }^{29}$ ) and the developed program. A ruler function was used to manually measure the diameters of NRs in an image segment, while the developed program and the commercial software performed an automated analysis. In the second step, human subjects and the commercial software applied the thresholding method to calculate the areal density ratio of NRs. The developed software calculated this ratio on the basis of information acquired during the first measurement: number and size of NRs.Table 1 summarizes the results of the validation procedure. Additionally, histograms and animations demon-

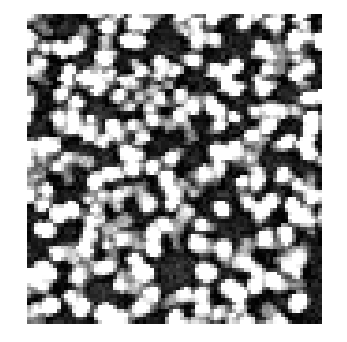

Fig. 4 Image used for verification purposes.

strating the image processing of SEM images of quantum dots and InP nanostructured thin-films are provided in the supplementary information.

\section{Results}

\subsection{Physical Characterization}

The AFM and XRD techniques were used to verify the surface morphology and provide the reference point for further analysis. The XRD pattern (Fig. 5) shows a dominating presence of vertically grown $\mathrm{Zn}(\mathrm{OH})_{2}$ crystals in (100) and (101) orientations. These orientations were in agreement with the 
surface images from AFM (Fig. 6), where $200 \mathrm{~nm}$ tall rod-like crystals were observed. The AFM images show clearly that the areal density and NRs size distribution varies according to location and a single image cannot be used to represent the whole sample.

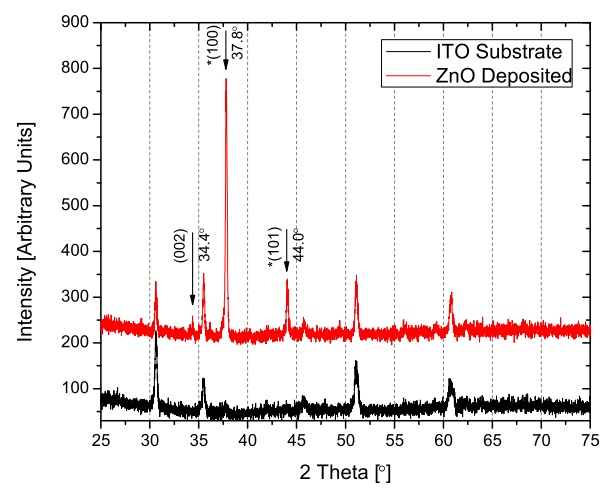

Fig. 5 XRD crystallographic analysis. XRD of grown $\mathrm{ZnO}$ on ITO/glass (red line) and its ITO/glass substrate (black line). Braggs angles and the corresponding crystal orientation for $\mathrm{ZnO}$ are noted. The $*(100)$ and $*(101)$ of the 2 theta angle correspond to $\mathrm{Zn}(\mathrm{OH})_{2}$ peaks.

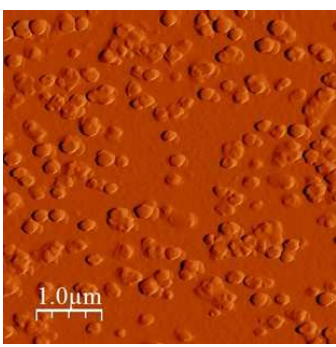

(a)

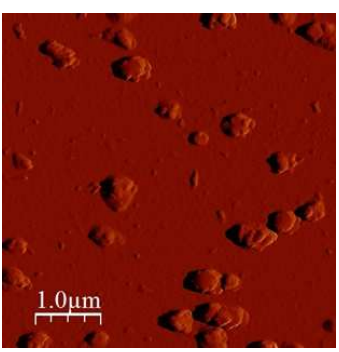

(c)

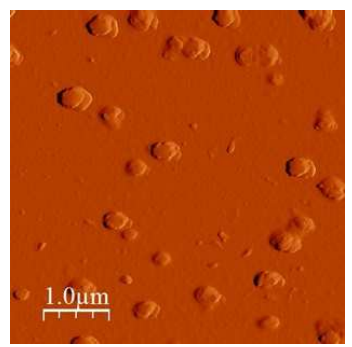

(b)

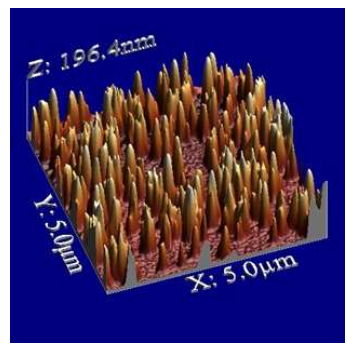

(d)
Fig. 6 Atomic force microscopy images of nanorods on ITO glass substrate: (a) water-air interface of the sample (location AFM1), (b) vertical midway along the sample (location AFM2), (c) the end of the sample (location AFM3), (d) 3-D representation of the atomic force micrograph from a location AFM1.

\subsection{Program implementation}

Fig. 7 shows the main steps of SEM image enhancement, while Fig. 8 presents the NR calculation steps performed by the developed software. Additional high quality processing animations are provided in supporting materials.

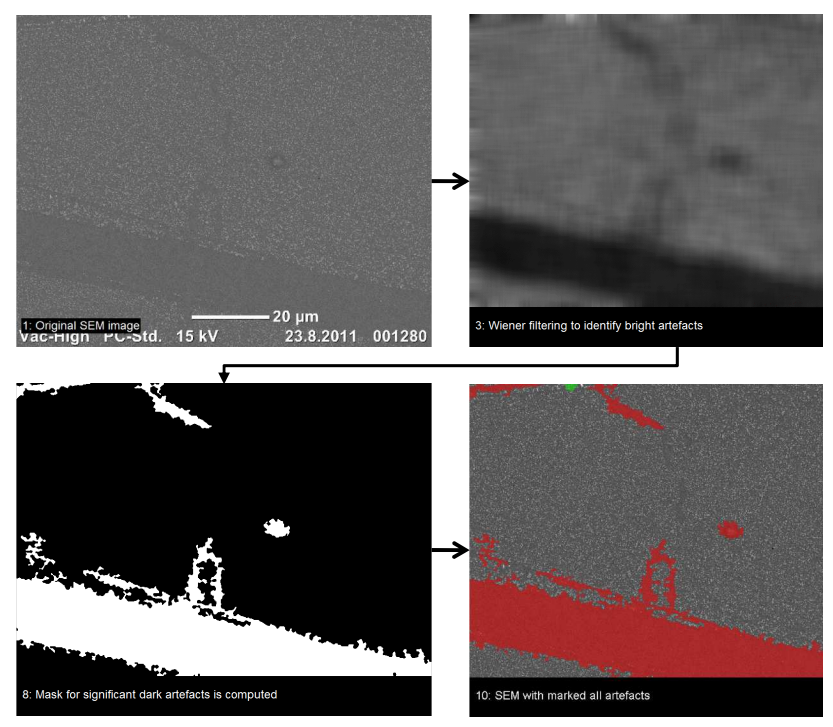

Fig. 7 The selected main steps of SEM images artefacts removal and enhancement processing: (1) Original SEM image, (3) Wiener filtering to identify bright artefacts, (8) Mask for significant dark artefacts is computed, (10) SEM with marked all artefacts.

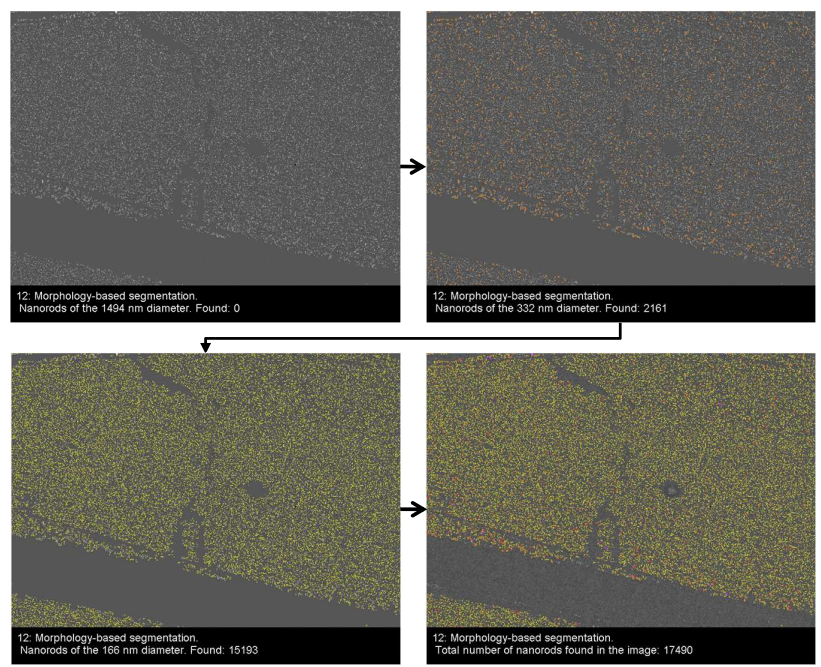

Fig. 8 The selected major steps of nanorods counting and areal density computation; the amount of NR with following diameter: 0 $(1494 \mathrm{~nm}), 2161(332 \mathrm{~nm}), 15193(166 \mathrm{~nm}), 17490$ (total amount of NR found in the image). 


\subsection{Areal density ratio}

Fig. 9 presents the average areal density of three strips, plotted as a function of distance from the water-air interface. For both the developed program and commercial Nikon software, it can be observed a decrease in the areal density ratio of NRs as the distance from the water-air interface increases to about 1.3 $\mathrm{mm}$. Beyond this range, the areal density ratio of NRs begins to increase steadily and saturates to $33 \%$ and $18 \%$, according to the developed program and Nikon software, respectively. In addition, Fig. 9 presents the areal density for each strip in the SEM images. As seen, areal density distributions at different locations of the sample differ significantly.

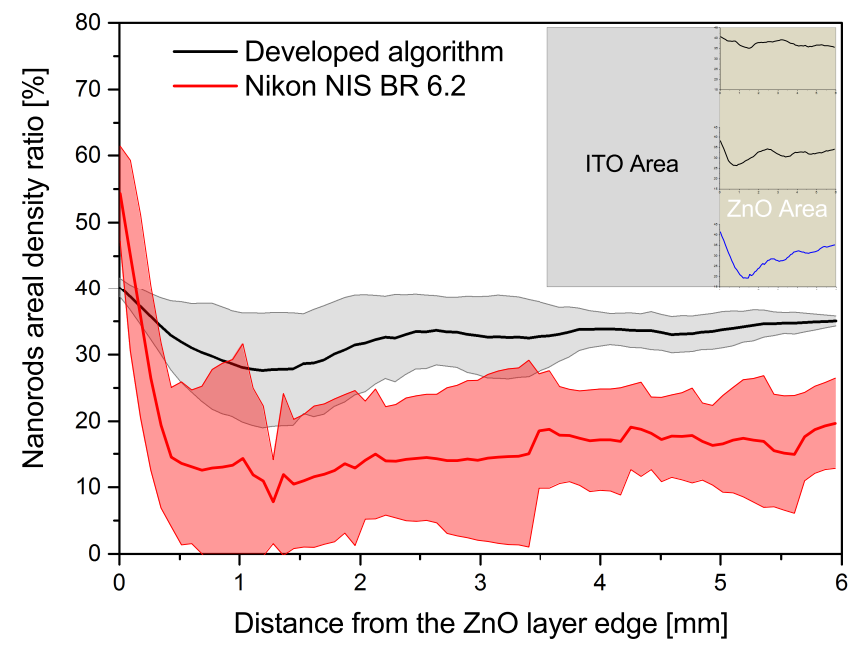

Fig. 9 Average of the nanorod areal density as a function of distance from the water-air interface. Additional plots in the upper right-hand corner (insert) present the areal density extracted from separate SEM image stripes.

\subsection{Diameter size distribution}

Fig. 10 is a logarithmic plot showing the number of NRs with distinct diameters based on data produced by the developed program. These diameters are separated into different ranges. The number of the smallest recognizable size $(166 \mathrm{~nm})$ is correlated with the image resolution and stands for the minimal recognizable structure of 2 pixels diameter. All measurement devices (SEM, AFM, etc.) provide a scaling line, which is used to calculate the area of the analysed image and the relation pixel/actual size: for $\mathrm{ZnO}$ SEM images 1 pixel $=83$ $\mathrm{nm}$. Accordingly, most of the NRs $(>80 \%)$ have a diameter in the range of $166 \mathrm{~nm}$ to $332 \mathrm{~nm}$, while the majority of the remaining structures range from 332 to $498 \mathrm{~nm}$ in diameter. The percentage of NRs with a diameter greater than $833 \mathrm{~nm}$ is practically non-existent and can be considered negligible.

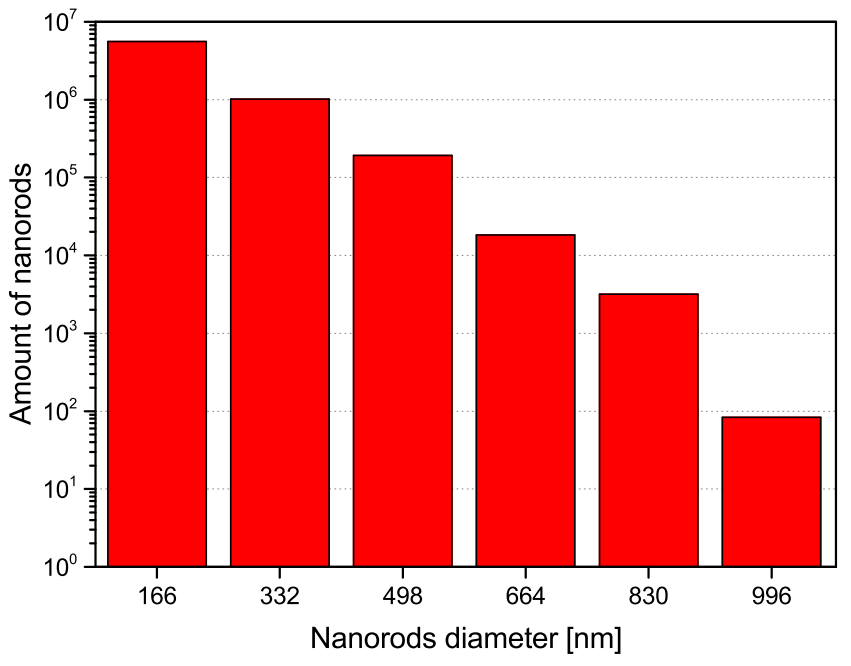

Fig. 10 Nanorod diameter size distribution.

The 3-D plot presented in Fig. 11, combines diameter size distribution data with distance from the water-air interface. An interesting trend, similar to that in Fig. 10, can be seen for NRs larger than $500 \mathrm{~nm}$. Moreover, 3-D figure shows that the whole film analyzed in this study consists largely of NRs with a diameter of less than half a micron.

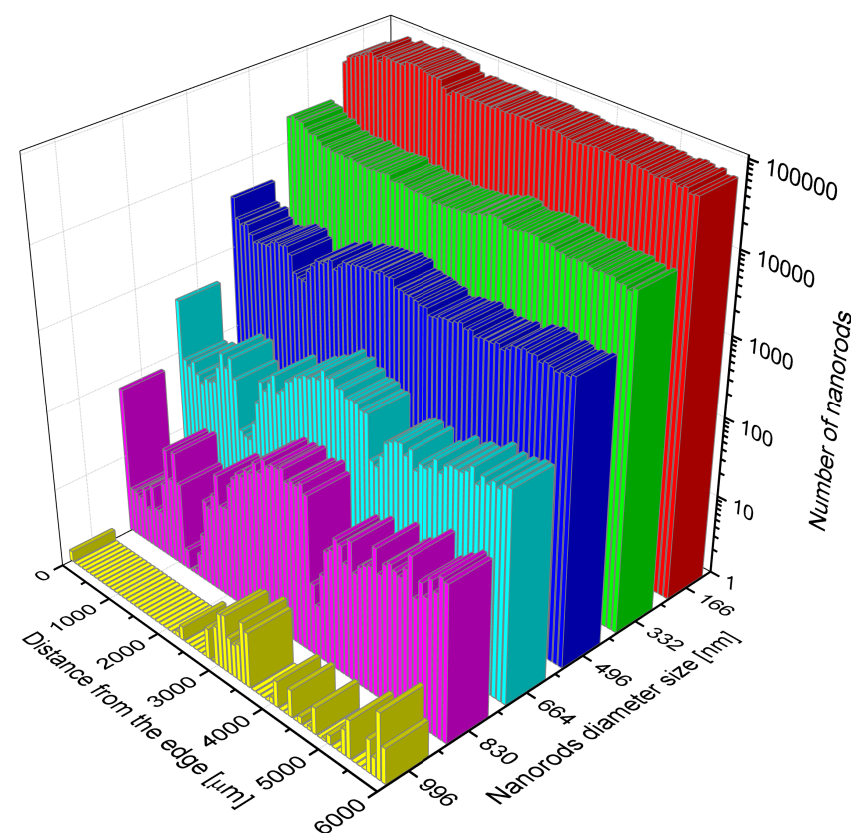

Fig. 11 Number of nanorods vs. NR diameter distribution and distance from the water-air interface. 


\subsection{Program verification}

A validation of the developed program against the manual reference and commercial software is depicted in Table 1. Additionally, validation based on the various SEM images is provided in the supplementary information.

Table 1 Comparison between manual and automated counting methods

\begin{tabular}{lcccc}
\hline & Subject 1 & Subject 2 & Nikon NIS-BR6.2 & Developed program \\
\hline Counted nanorods & 110 & 133 & 107 & 123 \\
Areal nanorods density ratio & 0.35 & 0.32 & 0.37 & 0.42 \\
\hline
\end{tabular}

\section{Discussion}

The main goal of this study is to present an automated software recognition approach to assess quantitative information on nanostructured thin-films. Differing from other deposition methods, the ECD method provides samples that are not perfectly uniform, and are, therefore, ideal for testing the capabilities of the developed software. Although the XRD plot (Fig. 5) shows a strong presence of $\mathrm{Zn}(\mathrm{OH})_{2}$ rather than $\mathrm{ZnO}$, the presence of vertically-oriented nanostructures, recognized by AFM and SEM, was critical for this investigation.

The advantage of image enhancement processing is particularly visible in Fig. 9, where results provided by the developed software are compared with the commercial solution. Although they all show a similar trend, a noticeable difference is present in areal density values. This divergence arises from the distinct methods employed by our program and the used commercial software. The former calculates areal density based on the size and number of NRs. It summarizes NRs of a specific diameter and calculates areal density on the basis of that information. The commercial software, on the other hand, applies a simple 'binarization and thresholding' method. Furthermore, although it considers scratches as areas without NR coverage, it does not subtract these areas from the total inspected image area. This results in a reduced NR areal density value. Our software pre-enhances images and analyses areal density excluding scratches and artefacts from the processing. Moreover, compared to the commercial software, it shows lower areal density variation (error lines), which is mostly attributed to artefact removal and a more precise calculating method.

As mentioned earlier, both plots show a decreasing trend in the surface area covered by NRs in the scanned region as the distance of the scanning location increases from the water-air interface. Additional plots in the upper-right hand corner of Fig. 9 reveal another interesting trend - the areal density of NRs also differs in the vertical axis along the sample. One likely mechanism for the uneven horizontal packing of NRs in the sample is geometric confinement due to water tension at the interface, making it more favourable for nucleation. In addition, the geometric configuration and size of the cathode produces an uneven electric field distribution ${ }^{30}$. Due to a slow diffusion rate, oxygen diffusion in particular reaches its highest value at the water interface, effectively increasing cathodic reactions ${ }^{31}$. Many groups have reported different electric field strengths in ECD leading to different growth mechanisms, which seem to influence deposition properties significantly $^{32-34}$. The presented software in this study provides evidence for further investigation and optimization of deposition processes. In Fig. 10, the logarithmic scale provides quantitative information about the size distribution of NRs in the analyzed area. This observation is in agreement with the physical characterization provided by AFM and XPS at different locations. It is worth noting that the developed software provides in-depth information on the size distribution of NRs, acquired from a significantly larger sample area. Thus, it provides more valuable information than a single imaging.

Associated with SEM image resolution, the images quality is the main limitation in this approach. This obstacle can be overcome by increasing image resolution or magnification. Of these options, the first one requires a more advanced SEM device, while the second solution requires a significantly larger number of images to investigate the same area. This brings us back to the fundamental principle of sampling frequency, namely the Shannon theorem, i.e., using a suitable accuracy that achieves the required task ${ }^{35}$. Regardless of the implemented solution, the developed software is capable of more precise processing, resulting in narrower NR size distribution ranges as demonstrated by analysis of additional structures.

A 3-D representation (Fig. 11) compiles all data acquired by the developed software and provides quantitative information about the size distribution of NRs on the sample surface. All NRs below $330 \mathrm{~nm}$ present a fairly even areal distribution along the sample surface, while the areal distribution of larger NRs becomes more uncertain with increasing diameter size.

To measure the usability of the developed algorithms, we performed a verification experiment on a small sample. The number of NRs counted by our software proved almost equal to the average value calculated by two human subjects, proving the validity of the achieved results. Differences in the areal density ratio of NRs arise from differences in the measurement method: our human subjects and the commercial software used the thresholding method, while the developed program calculated the number of NRs and their diameter. Since the number of NRs calculated by the developed software has been proven correct, it should describe more precisely the area occupied by NRs than the simple thresholding method.

Although this implementation is based on analyzing the hexagonal shape of nanostructures, it can be easily applied to nanostructures of various shapes by implementing enhanced 
recognition features. This unique approach allows a precise determination of the areal density and size distribution of nanostructures over a significantly larger area than current approaches. Combining the functionality of the developed program with a surface characterizing unit such as SEM or AFM and automating it (via programming) would help to reach the desired goal: improved quantitative characterization of nanostructured thin-films, critical for nanoscale devices.

\section{Conclusions}

This study presents an autonomous characterization system capable of extracting quantitative information on the areal density and size distribution of fabricated nanostructures. This unique approach allows a precise determination of the areal density and size distribution of nanostructures over a significantly larger area than current approaches. Combining the functionality of the developed program into a surface characterising unit such as SEM or AFM and automating it (via programming) would help to reach the desired goal: improved quantitative characterization of nanostructured thin-films, a critical for nanoscale devices.

\section{Acknowledgements}

The authors thank to dr. Bell Gavin from the University of Warwick, for providing one of the verification images.

\section{References}

1 Y. Xia, P. Yang, Y. Sun, Y. Wu, B. Mayers, B. Gates, Y. Yin, F. Kim and H. Yan, Advanced Materials, 2003, 15, 353-389.

2 One-Dimensional Nanostructures, ed. Z. M. Wang, Springer, 2008.

3 D. Moore and Z. L. Wang, J. Mater. Chem., 2006, 16, 3898-3905.

4 L. Chow, O. Lupan, G. Chai, H. Khallaf, L. Ono, B. R. Cuenya, I. Tiginyanu, V. Ursaki, V. Sontea and A. Schulte, Sensors and Actuators A: Physical, 2013, 189, $399-408$.

5 W. L. Leong and J. J. Vittal, Chemical Reviews, 2011, 111, 688-764.

6 S. Xu and Z. Wang, Nano Research, 2011, 4, 1013-1098.

7 L. Li, T. Zhai, Y. Bando and D. Golberg, Nano Energy, 2012, 1, 91 - 106.

8 O. Velev, P. Tessier, A. Lenhoff and E. Kaler, Nature, 1999, 401, 548-548.

9 B. El Zein, S. Boulfrad, G. Jabbour and E. Dogheche, Applied Surface Science, 2014, 292, 598-607.

10 Y. Kim, J. Zhu, B. Yeom, M. Di Prima, X. Su, J.-G. Kim, S. J. Yoo, C. Uher and N. A. Kotov, Nature, 2013, 500, 59-63.
11 C. J. Lee, T. J. Lee, S. C. Lyu, Y. Zhang, H. Ruh and H. J. Lee, Applied Physics Letters, 2002, 81, 3648-3650.

12 K. Hiruma, M. Yazawa, T. Katsuyama, K. Ogawa, K. Haraguchi, M. Koguchi and H. Kakibayashi, Journal of Applied Physics, 1995, 77, 447-462.

13 C. Geng, Y. Jiang, Y. Yao, X. Meng, J. A. Zapien, C. Lee, Y. Liftshitz and S. Lee, Advanced Functional Materials, 2004, 14, 589-594.

14 J. Krc, M. Zeman, O. Kluth, F. Smole and M. Topic, Thin Solid Films, 2003, 426, 296 - 304.

15 L. C. Gontard, D. Ozkaya and R. E. Dunin-Borkowski, Ultramicroscopy, 2011, 111, $101-106$.

16 A. Virga, P. Rivolo, E. Descrovi, A. Chiolerio, G. Digregorio, F. Frascella, M. Soster, F. Bussolino, S. Marchi, F. Geobaldo and F. Giorgis, Journal of Raman Spectroscopy, 2012, 43, 730-736.

17 B. Liu and E. S. Aydil, Journal of the American Chemical Society, 2009, 131, 3985-3990.

18 H. Huang, Y. Lee, C. Chow, C. Ong, M. Tse and O. Tan, IEEE Sensors, 2008, pp. $114-117$.

19 S. Mondini, A. M. Ferretti, A. Puglisi and A. Ponti, Nanoscale, 2012, 4, 5356-5372.

20 A. H. Roeder, A. Cunha, M. C. Burl and E. M. Meyerowitz, Development, 2012, 139, 3071-3080.

21 P. Soille, Morphological Image Analysis; Principles and Applications, Springer-Verlag Telos, 2007.

22 P. Ravirajan, A. M. Peiro, M. K. Nazeeruddin, M. Graetzel, D. D. C. Bradley, J. R. Durrant, J. Nelson and A. P. Fe, Methods, 2006, 110, 76357639.

23 L. Baeten, B. Conings, H.-G. Boyen, J. D'Haen, A. Hardy, M. D'Olieslaeger, J. V. Manca and M. K. Van Bael, Advanced Materials, 2011, 23, 2802-2805.

24 I. Gonzalez-Valls and M. Lira-Cantu, Energy Environ. Sci., 2009, 2, 19 34.

25 B. Seipel, A. Nadarajah, B. Wutzke and R. Kainenkamp, Materials Letters, 2009, 63, $736-738$.

26 C. V. D'Alkaine and M. N. Boucherit, Journal of The Electrochemical Society, 1997, 144, 3331-3336.

27 F. Jin, P. Fieguth, L. Winger and E. Jernigan, International Conference on Image Processing, 2003.

28 N. Otsu, Systems, Man and Cybernetics, IEEE Transactions on, 1979, 9, 62-66.

29 Nikon, http://www.nis-elements.com/.

30 J. A. H. H.R. Thirsk, Guide to the Study of Electrode Kinetics, Academic Press Inc, May 1972.

31 A. Goux, T. Pauporte and D. Lincot, Electrochimica Acta, 2006, 51, 3168 -3172 .

32 H. E. Prakasam, K. Shankar, M. Paulose, O. K. Varghese and C. A. Grimes, The Journal of Physical Chemistry C, 2007, 111, 7235-7241.

33 B. Melody, T. Kinard and P. Lessner, Electrochemical and Solid-State Letters, 1998, 1, 126-129.

34 Y.-M. Li and L. Young, Journal of The Electrochemical Society, 2001, 148, B337-B342.

35 C. Shannon, Proceedings of the IEEE, 1984, 72, $1192-1201$. 